\title{
Schizophrenia-associated SLC39A8 polymorphism is a loss-of-function allele altering glutamate receptor and innate immune signaling
}

Wei Chou Tseng ${ }^{1}$, Veronica Reinhart ${ }^{2}$, Thomas A. Lanz $\mathbb{1}^{3}$, Mark L. Weber ${ }^{3}$, Jincheng Pang ${ }^{3}$, Kevin Xuong Vinh Le ${ }^{3}$, Robert D. Bell ${ }^{3}$, Patricio O'Donnell (1) ${ }^{4}$ and Derek L. Buhl (i) ${ }^{4}$

\begin{abstract}
Schizophrenia is a complex and heterogenous disease that presents with abnormalities in glutamate signaling and altered immune and inflammatory signals. Genome-wide association studies have indicated specific genes and pathways that may contribute to schizophrenia. We assessed the impact of the functional missense variant SLC39A8 (ZIP8)-A391T (ZIP8 ${ }^{\text {A391T }}$ ) on zinc transport, glutamate signaling, and the neuroinflammatory response. The ZIP8 ${ }^{\text {A391T }}$ mutation resulted in reduced zinc transport into the cell, suggesting a loss in the tight control of zinc in the synaptic cleft. Electrophysiological recordings from perturbed neurons revealed a significant reduction in NMDA- and AMPAmediated spontaneous EPSCs (sEPSCs) and a reduction in GluN2A and GluA1/2/3 receptor surface expression. All phenotypes were rescued by re-expression of wild-type ZIP8 (ZIP8 ${ }^{\mathrm{WT}}$ ) or application of the membrane-impermeable zinc chelator ZX1. ZIP8 reduction also resulted in decreased BBB integrity, increased IL-6/IL-1 $\beta$ protein expression, and increased NFKB following TNFa stimulation, indicating that ZIP8 loss-of-function may exacerbate immune and inflammatory signals. Together, our findings demonstrate that the A391T missense mutation results in alterations in glutamate and immune function and provide novel therapeutic targets relevant to schizophrenia.
\end{abstract}

\section{Introduction}

Recent genome-wide association studies (GWAS) have improved our understanding of the genetic basis of psychiatric disorders ${ }^{1}$. Several independent GWAS studies have revealed genetic associations between schizophrenia and functional missense variants in the SLC39A8 gene $^{2-5}$, which encodes the cation metal transporter ZIP8 ${ }^{6}$. Among these human single nucleotide polymorphism (SNP) variants, non-synonymous rs13107325 presents an interesting mechanism as it encodes an amino acid change from alanine to threonine in the ZIP8 transporter at position $391\left(\mathrm{ZIP}^{\mathrm{A} 391 \mathrm{~T}}\right)^{2}$. ZIP8 belongs to the Solute Carrier Family and transports divalent metal ions, including zinc and manganese, from the extracellular

Correspondence: Derek L. Buhl (derek.buhl@gmail.com)

${ }^{1}$ Wave Life Sciences, Cambridge, MA 02138, USA

${ }^{2}$ Biogen, Cambridge, MA 02139, USA

Full list of author information is available at the end of the article environment or intracellular compartments into the cytosol $^{6,7}$. Despite the strong genetic association between ZIP8 and schizophrenia, the role of ZIP8 and its variants in schizophrenia pathophysiology have remained largely uncharacterized.

Zinc, in its free ionic form $\mathrm{Zn}^{2+}$, is indispensable for all systems throughout the body, especially for immune and nervous system function. A growing body of evidence has indicated that the level of zinc is highly regulated due to its dynamic role in brain physiology. Zinc is incorporated into presynaptic vesicles via a variety of zinc transporters enriched at glutamatergic nerve terminals found in cerebral cortex, amygdala, cingulate cortex, hippocampus, and olfactory bulb ${ }^{8,9}$. At these synapses, zinc is coreleased with glutamate into the synaptic cleft, transiently bringing interneuronal zinc concentration to micromolar levels ${ }^{10-12}$. This extracellular zinc profoundly affects the activity of a variety of postsynaptic ionotropic receptors,

\section{(c) The Author(s) 2021}

(c) (i) Open Access This article is licensed under a Creative Commons Attribution 4.0 International License, which permits use, sharing, adaptation, distribution and reproduction cc) in any medium or format, as long as you give appropriate credit to the original author(s) and the source, provide a link to the Creative Commons license, and indicate if changes were made. The images or other third party material in this article are included in the article's Creative Commons license, unless indicated otherwise in a credit line to the material. If material is not included in the article's Creative Commons license and your intended use is not permitted by statutory regulation or exceeds the permitted use, you will need to obtain permission directly from the copyright holder. To view a copy of this license, visit http://creativecommons.org/licenses/by/4.0/. 
and among these targets of synaptic zinc, $N$-methyl-Daspartate receptors (NMDARs) are rather unique as they display very high sensitivity to extracellular zinc ${ }^{13,14}$. In particular, the primary synaptic NMDAR subunit, GluN2A, is inhibited by nanomolar levels of zinc via an allosteric binding site located in the large bilobate $\mathrm{N}$ terminal domain of the GluN2A subunit ${ }^{15}$. The result of zinc-mediated inhibition of GluN2A-NMDARs leads to a decrease in calcium influx and thus a decrease in neuronal activation ${ }^{13,16}$. Glutamate hypofunction in the prefrontal cortex has been implicated in cognitive dysfunction associated with many brain disorders, including schizophrenia, which is supported by the finding that numerous drugs blocking NMDAR receptors are known to trigger acute psychotic reactions ${ }^{17,18}$. Thus, alterations in zinc signaling may contribute to synaptic anomalies in schizophrenia.

In addition to the role zinc plays in regulating synaptic activity, zinc homeostasis is also important for blood-brain barrier (BBB) integrity and immune response, both of which are proposed to significantly influence many neurological conditions. In schizophrenia, disruption of the $\mathrm{BBB}$ and exacerbated immune responses are thought to contribute to the progression and cognitive symptoms of the disease (for review, see ref. ${ }^{19}$ ). Recent evidence from a PheWAS study indicates that the ZIP8 ${ }^{\mathrm{A} 391 \mathrm{~T}}$ missense variant is associated with intracranial hemorrhage and cerebrovascular disease ${ }^{20}$, suggesting that ZIP8 may play a role in the BBB.

Despite the strong genetic association between $\mathrm{ZIP}^{\mathrm{A} 391 \mathrm{~T}}$ and schizophrenia, the role of ZIP8 and its variants in schizophrenia have remained largely uncharacterized. Here, we report that the ZIP8 ${ }^{\mathrm{A} 391 \mathrm{~T}}$ mutation is a loss-of-function allele that leads to changes in glutamate receptor expression and function, likely through decreased zinc import at the synapse. We further provide evidence that replacing the ZIP8 ${ }^{\mathrm{A} 391 \mathrm{~T}}$ mutation with wildtype ZIP8 (ZIP8 ${ }^{\mathrm{WT}}$ ) or removal of the excess zinc at the synapse resolves these deficits. In addition, we show that excessive zinc results in elevated neuroinflammatory responses both in vitro and in vivo. As emerging evidence strengthens the link between neuroinflammation and glutamate hypofunction in psychiatric disorders ${ }^{21,22}$, this study provides a potential disease mechanism of schizophrenia that includes both of these phenomena, suggesting a novel direction for developing future therapeutics targeting schizophrenia.

\section{Materials and methods}

All experiments involving animals in this study were carried out in accordance with the recommendations of the Guide for the Care and Use of Laboratory Animals. The protocol was approved by the Pfizer Institutional Animal Care and Use Committee.

\section{DNA constructs and antibodies}

Constructs overexpressing human ZIP8 ${ }^{W T}, Z I P 8^{A 391 T}$, $Z I P 8^{W T}-m C h e r r y$, or ZIP8 ${ }^{A 391 T}-m C h e r r y$ and all lentiviral vectors expressing $L u c$-Gfp-shRNA or Zip8-Gfp-shRNA were synthesized by Genscript, Inc. Plasmid DNA was transfected into Chinese hamster ovary $(\mathrm{CHO})$ cells or primary pyramidal neurons by Lipofectamine 3000 or Lipofectamine LTX, respectively (Thermo Fisher Scientific Inc.) according to the manufacturer's protocol. Primary antibodies include rabbit anti-GluA1 (PC246, Millipore), rabbit anti-GluN1(AGC-001, Alomone labs), rabbit anti-GluN2A (AGC-002, Alomone labs), rabbit anti-GluN2B (AGC-003, Alomone labs), rabbit antiGluA2/3 (AB1506, Millipore). Secondary antibody Alexa Fluor 568 was purchased from Thermo Fisher Scientific.

\section{Zinc import into $\mathrm{CHO}$ cells}

The zinc import assay used in the present study was adapted from ref. ${ }^{23}$. Briefly, CHO cells cultured on 6-well plates were transfected with either ZIP8 ${ }^{W T}-m$ Cherry or $Z I P 8^{A 391 T_{-}}$- $C$ Cherry using Lipofectamine 3000 from Thermo Fisher Scientific. Two days before imaging, cells were plated onto $35 \mathrm{~mm}$ glass-bottom culture dishes containing $2 \mathrm{~mL}$ of growth medium. Cells were incubated in fresh growth medium with $5 \mu \mathrm{M} \mathrm{ZP1}$ or DMSO and $4 \mu \mathrm{M}$ Hoechst $332580.5 \mathrm{~h}$ before imaging. Cells were then washed twice with DMEM without phenol red, and $2 \mathrm{~mL}$ of serum-free DMEM containing $20 \mu \mathrm{M} \mathrm{ZnCl}$ : pyrithione was applied at a ratio of 1:2. Cells overexpressing mCherry were imaged with live microscopy every $10 \mathrm{~min}$ for $60 \mathrm{~min}$ to monitor changes in green fluorescence signal. As no differences were observed between cell groups at the first imaging timepoint $(T 0)$, all other timepoints were normalized to each cell's $T 0$ to evaluate differences in $\mathrm{ZnCl}_{2}$ influx. Signal mean/standard deviation of each timeframe was analyzed using ImageJ. In determining the membrane targeting of $Z I P 8^{W T}$-mCherry or ZIP8 $8^{A 391 T}$-mCherry construct, the cell expressing either construct was profiled using ImageJ to extract fluorescence signal across the cell while avoiding intracellular organelles such as nucleus and Golgi. The ratio of membrane/cytosol signal was quantified from ten cells per condition, and one representative trace of each condition is shown in Fig. 1C.

\section{Immunofluorescence staining}

Rat pyramidal neurons were isolated and cultured on cover slips and then transfected with lentivirus encoding either Luc-Gfp-shRNA or Zip8-Gfp-shRNA at DIV7. At DIV28-30, cover slips were washed twice with conditioned culture media (Neurobasal, B27, and Glutamax) before the incubation with $100 \mu \mathrm{M} \mathrm{ZX1}$ at $37^{\circ} \mathrm{C}$ for 0,30 , or $180 \mathrm{~min}$. During the last $30 \mathrm{~min}$ of incubation, primary antibodies against different glutamate receptor subunits 
were added to the cover slip at $37^{\circ} \mathrm{C}$ for $30 \mathrm{~min}$. All cover slips were then washed three times with conditioned culture media and incubated with secondary Alexa fluor 568 antibody 1:100 (Life Technologies) at $37^{\circ} \mathrm{C}$ for $30 \mathrm{~min}$. Cover slips were then washed three times with PBS, fixed with $4 \%$ paraformaldehyde/ $4 \%$ sucrose at room temperature for $15 \mathrm{~min}$, and then mounted with ProLong Gold solution (Thermofisher). Confocal microscopy was performed using a Zeiss inverted microscope. All images were analyzed and quantified using custom Matlab scripts (code available upon request).

\section{Patch clamp of rat cortical culture}

Rat fetal (E18) frontal cortex primary neurons were grown on 12-mm round poly-D-lysine-coated cover slips. Neurons were transfected with shRNA with or without rescue ZIP8 plasmids using Lipofectamine 3000 (Thermo Fisher) at DIV7 following the manufacturer's protocol. At DIV28-30, cover slips with primary neurons were used for patch clamp studies investigating $\alpha$-amino3-hydroxy-5-methyl-4-isoxazolepropionic acid receptor (AMPAR)- and NMDAR-mediated glutamatergic spontaneous activity and miniature excitatory postsynaptic currents (mEPSCs) as described previously ${ }^{24}$. In brief, $100 \mu \mathrm{M}$ bicuculline was added to the external solution to block $\gamma$-aminobutyric acid $(\mathrm{GABA})_{\mathrm{A}}$ receptors throughout the recording. After break-in, 2 min of activity was recorded until a stable state was reached. (2R)-amino-5phosphonovaleric acid (APV) $(50 \mu \mathrm{M})$ was then applied for 5-6 min to block NMDARs and isolate AMPA EPSCs, followed by wash out from external buffer with bicuculline. Conversely, $10 \mu \mathrm{M}$ 6-cyano-7-nitroquinoxaline-2,3-dione (CNQX) was then applied for another 5-6 min to block AMPARs and isolate NMDA EPSCs, followed by the application of both APV and CNQX to get baseline activity (Fig. S1D). For mEPSC recordings, $1 \mu \mathrm{M}$ tetrodotoxin (TTX) was added to the external solution to block neuronal firing in addition to the sequence of APV and CNQX as above to acquire AMPAand NMDA-mediated mEPSCs, respectively. Baseline activity of mEPSCs was obtained by the application of both APV and CNQX at the end of each recording (Fig. $\mathrm{S} 1 \mathrm{E})$. All mEPSC recordings were analyzed by Mini Analysis Program (Synaptosoft). Detection parameters were set as follows: threshold at 1 , period to search a local maximal at $80 \mathrm{~ms}$, time before a peak for baseline at $20 \mathrm{~ms}$, period to search a decay at $40 \mathrm{~ms}$, fraction of peak to find a decay time at 0.37 , period to average a baseline at $10 \mathrm{~ms}$, and area threshold at 2. Cells were rejected from analysis if the series resistance was greater than $15 \mathrm{M} \Omega$ after membrane rupture. Each event was then examined manually to ensure the accuracy of detection.

\section{Mice surgery and slice electrophysiology}

CA1 neuronal cell bodies were labeled by bilateral stereotaxic injection of 6-8-week-old male C57/B6 mice with lentiviral vectors encoding either $L u c-G f p$ shRNA or Zip8-Gfp-shRNA at the ventral hippocampus (bregma $-3.4 \mathrm{~mm} ; X \pm 3.35 \mathrm{~mm} ; Z-3.8$ and $-3.4 \mathrm{~mm}$, $0.5 \mu \mathrm{L} / \mathrm{z}$ ). Lentiviral vectors were purchased from Origene. Mice surgeries, the preparation of acute brain slices, and the setup of the recording system were conducted according to a previously published study ${ }^{25}$. The detailed protocol for Schaffer collateral stimulation was performed as previously reported ${ }^{26}$. Patch pipettes with 3-5 M $\Omega$ resistances were filled with internal solution. Cells were rejected from analysis if the series resistance was greater than $30 \mathrm{M} \Omega$ after membrane rupture.

\section{Biotinylation and Western blot}

Rat pyramidal neurons were isolated and cultured on 6well dishes and then transfected with lentivirus encoding either Luc-Gfp-shRNA or Zip8-Gfp-shRNA at DIV7. At DIV28-30, biotinylated lysates were prepared and blotted according to a published protocol ${ }^{27}$. After primary antibody incubation, blots were developed using a Typhoon imaging system (GE Healthcare) and the band intensity was quantified using ImageJ.

\section{Lipopolysaccharide challenge and measurement of cytokines}

Male B6:129S Zip8 ${ }^{+/-}$mice and age-matched wild-type mice (6-17 weeks) were given a first $2.5 \mathrm{mg} / \mathrm{kg}$ dose of lipopolysaccharides (LPS) by intraperitoneal injection, followed by a second injection $6 \mathrm{~h}$ later, and a third injection $24 \mathrm{~h}$ after the first injection. An intravenous retro-orbital injection of $0.1 \mathrm{~mL} 70 \mathrm{kDa}$ dextran-488 (Thermo Fisher) was given $28 \mathrm{~h}$ after the first LPS injection. After $30 \mathrm{~min}$, each animal was euthanized, and the brain was collected and bisected for biochemistry and histopathological analysis. Blood $(5 \mathrm{~mL})$ was drawn and serum was isolated and tested in Meso Scale Discovery assays according to the manufacturer's protocol to analyze the levels of various cytokines. Brain homogenate was prepared by adding 5 volumes of $20 \mathrm{mM}$ Tris- $\mathrm{HCl}$ buffer ( $\mathrm{pH}$ 7.6) with a protease inhibitor cocktail and sonicating with a probe. FITC signal from the dextran injection was measured using a spectrometer and normalized by total protein concentration determined by BCA analysis.

\section{NFKB activation by TNFa stimulation}

HEK293 cells stably expressing a $3 \times \mathrm{xF}_{\mathrm{N}} \mathrm{B}$ reporter coupled to luciferase were generated in house. The day 
before transfection, HEK-NFKB cells were seeded onto 6 -well plate at 500,000 cells per well. The next day, $2 \mu \mathrm{g}$ of plasmids encoding different ZIP8 transcripts were transfected into HEK-NFKB cells using Lipofectamine 2000 following the manufacturer's protocol with a 3:1 ratio of lipid to DNA. After $24 \mathrm{~h}$, the cells were reseeded onto poly-D-lysine-coated clear-bottom 96-well plates at 50,000 cells per well. On the following day, the growth medium was replaced with $50 \mu \mathrm{L}$ of medium containing various amounts of TNF $\alpha(1,3,10$, $30,100$, or $300 \mathrm{ng} / \mu \mathrm{L})$. After $6 \mathrm{~h}$ of incubation, $50 \mu \mathrm{L}$ of $2 \times$ One Glo (Promega) was prepared and added to each well. The plate was incubated in the dark for $10 \mathrm{~min}$ prior to the readout of luminescence using Envision (Perkin Elmer).

\section{Results}

The ZIP8 ${ }^{A 391 T}$ SNP associated with schizophrenia patients in GWAS studies is a loss-of-function allele

To determine the nature of the human SNP rs13107325 variant encoding an A391T change in ZIP8, we generated human $Z I P 8^{W T}$ and $Z I P 8^{A 391 T}$ cDNA constructs tagged with mCherry and overexpressed them in $\mathrm{CHO}$ cells, a commonly used cell line for metal ion transport studies. Similar membrane localization was observed in cells overexpressing either $Z I P 8^{W T}-m$ Cherry or $Z I P 8^{A 391 T}$. $m$ Cherry construct, indicating that A391T mutation did not alter the membrane targeting of ZIP8 (Fig. 1). Cells were loaded with ZP1 green fluorescing dye $\mathrm{e}^{23}$ and liveimaged following the addition of $\mathrm{ZnCl}_{2}$ ions to the medium. The observation of increased green fluorescence signal into the cells was an expected result from overexpression of a functional ZIP8 transporter. In $\mathrm{CHO}$ cells overexpressing $Z I P 8^{W T}-m$ Cherry, we observed the expected time-dependent increase $(25 \%$ at $40 \mathrm{~min})$ of green fluorescence signal (Fig. 1A, B), indicating the robust import of $\mathrm{ZnCl}_{2}$ into the cytosol where it conjugates with ZP1 dye and emits green fluorescence. Cells overexpressing $Z I P 8^{A 391 T}$ - $m$ Cherry, however, had a much slower and smaller increase $(5 \%$ at $60 \mathrm{~min})$ of the $\mathrm{ZP} 1-\mathrm{ZnCl}_{2}$ fluorescence signal, suggesting an impairment of $\mathrm{ZnCl}_{2}$ import as a result of the A391T mutation. Importantly, we did not observe any differences in cell size or membrane targeting of ZIP8 ${ }^{\mathrm{WT}}$ or ZIP8 ${ }^{\mathrm{A} 391 \mathrm{~T}}$ (Fig. 1D, C). These data indicate that the SNP rs13107325 is a loss-of-function mutation in the ZIP8 transporter.

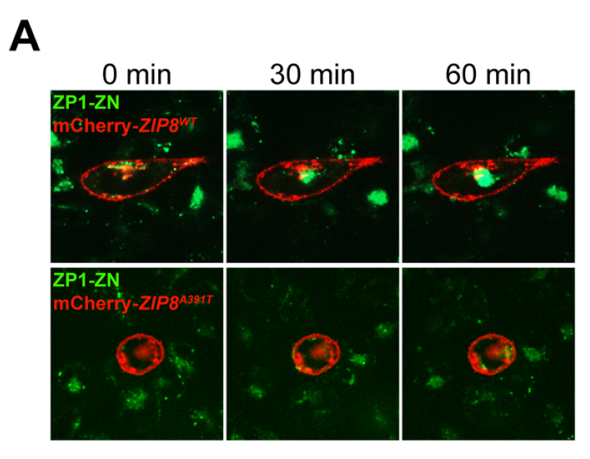

B

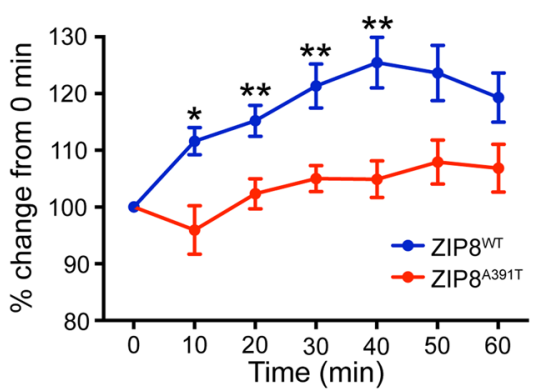

C
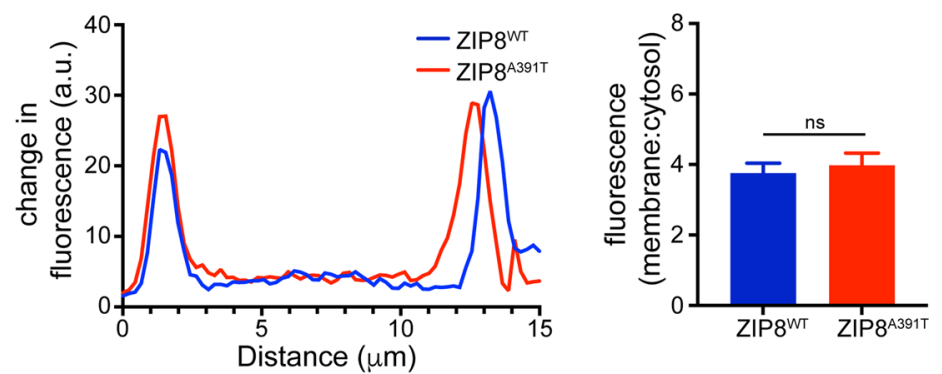

Fig. 1 Human ZIP8 $^{A 391 T}$ SNP associated with schizophrenia is a loss-of-function allele. A Time course of ZP1 signal after addition of $\mathrm{ZnCl}_{2}$ in CHO cells overexpressing either ZIP8 ${ }^{W T}$ or ZIP8 with the human SNP mutation (ZIP $8^{A 391 T}$ ) tagged with mCherry. B Quantification of GFP signals over time relative to $0 \mathrm{~min}$ for both $\mathrm{ZIP} 8^{\mathrm{WT}}$ - and ZIP8 $8^{\mathrm{A} 391 \mathrm{~T}}$-overexpressing $\mathrm{CHO}$ cells. The baseline measurements (T0) of ZIP8 ${ }^{\mathrm{WT}}$ and ZIP8 ${ }^{\mathrm{A} 391 \mathrm{~T}}$ were not significantly different (unpaired $t$-test, $P=0.11$ ). Compared to $\mathrm{ZIP} 8^{\mathrm{WT}}$, ZIP8 ${ }^{\mathrm{A} 391 \mathrm{~T}}$-expressing $\mathrm{CHO}$ cells had significantly lower $\mathrm{ZnCl}_{2}$ influx at multiple timepoints. $P=0.003$ (two-way ANOVA, $n=25-32$ per condition). Bonferroni's post-hoc test revealed significant differences at 10, 20, 30, and 40 min $\left({ }^{*} P=0.02,{ }^{* *} P<0.01\right)$. Error bars, SEM. C, D Overexpression of ZIP8 ${ }^{\mathrm{WT}}$ or ZIP8 ${ }^{\mathrm{A} 391 \mathrm{~T}}$ did not alter cell size (representative traces of cell size measurements shown in C). D Quantification of the membrane:cytosol signal. Ten cells from each group were used for quantification. Error bars, SEM. 

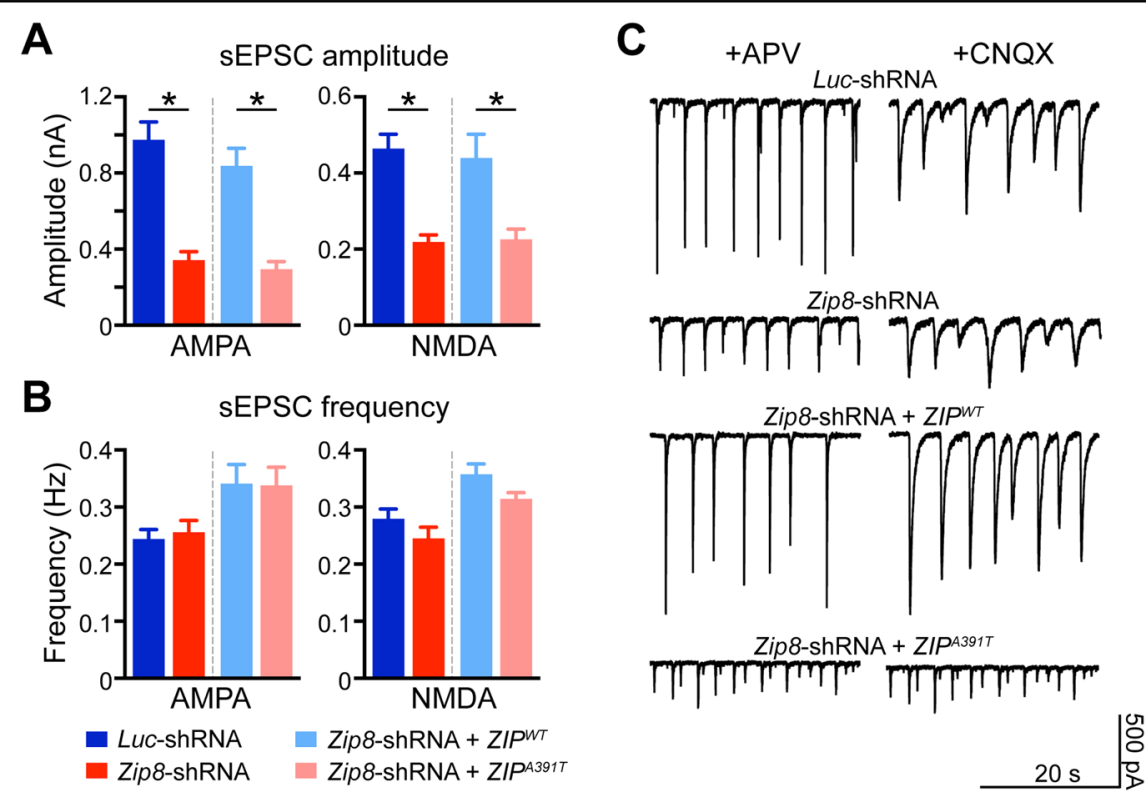

Fig. 2 ZIP8 total knockout (KO) and loss-of-function allele A391T displays neuronal activity-dependent deficits in glutamate receptormediated sEPSCs. A, B Quantification of sEPSC amplitude (A) and frequency (B) recorded from cultured D28-30 neurons. ${ }^{*} P<0.05$ (Student's $t$-test, $n=6-10$ per group). Error bars, SEM. C Representative raw traces of glutamate receptor-mediated sEPSCs in cultured pyramidal neurons expressing either Luc-shRNA, Zip8-shRNA, Zip8-shRNA + ZIP8 ${ }^{W T}$ plasmid, or Zip8-shRNA + ZIP8A391T plasmid. Note the significant loss of sEPSC amplitude, not frequency, in the presence of the ZIP8 ${ }^{A 391 T}$ mutation, consistent with the loss-of-function observed in Fig. 1. No changes were observed in mEPSC amplitude or frequency (Fig. S1).

Zinc is known to be an allosteric inhibitor of NMDA receptors ${ }^{16}$. Given the evidence for glutamate hypofunction as a major pathology in schizophrenia and that glutamate receptors are inhibited by excess zinc at axon terminals upon neuronal activity, we addressed the role of zinc import by ZIP8 in neurotransmission by using short hairpin RNA (shRNA) lentivirus targeting Zip8 or Luciferase in cultured rat hippocampal neurons. Neurons transfected with a hairpin targeting Zip8 3 weeks prior to recording (Zip8-shRNA) resulted in 90\% knockdown (KD) of Zip8 mRNA expression. Whole-cell patch clamp revealed significantly smaller amplitudes of both AMPAR- and NMDAR-mediated spontaneous excitatory postsynaptic currents (sEPSCs) in Zip8-shRNA neurons compared to Luc-shRNA transfected controls (Fig. 2A, C). The reduction of sEPSC amplitude in cultured neurons was rescued by co-expressing ZIP8 ${ }^{W T}$ but not ZIP8 ${ }^{A 391 T}$ construct (Fig. 2A, C), consistent with our finding that $Z I P 8^{A 391 T}$ is a loss-of-function allele that disrupts the import of synaptic zinc. sEPSC frequency was not affected by either ZIP8 ${ }^{W T}$ or ZIP8 ${ }^{A 391 T}$ transfection (Fig. $2 \mathrm{~B})$. These results support the hypothesis that ZIP8 hypofunction may lead to decreased zinc import and thus increased synaptic zinc and zinc-mediated AMPAR and NMDAR inhibition.

To ensure we were observing solely a postsynaptic effect, we next applied TTX to eliminate major neuronal activity in the culture and recorded mEPSCs caused by quantal release of presynaptic vesicles independent of stimulation in presynaptic termini. Interestingly, we did not observe a difference between ZIP8 KD and rescued neurons in terms of either mEPSC amplitude or frequency (Fig. S1A-C), suggesting that the deficit in sEPSCs recorded without TTX is activity dependent. Given that zinc can be co-released with glutamate at places such as hippocampal mossy fiber synapses ${ }^{28}$, it is likely that only during a firing event could zinc concentration rise to the levels required for inhibition of postsynaptic glutamate receptors ${ }^{16}$ in the synaptic cleft.

\section{ZIP8 knockdown leads to decreased surface localization of glutamate receptor subunits}

To evaluate whether the reduced amplitude of glutamate receptor-mediated sEPSCs is due to decreased surface localization of postsynaptic glutamate receptors, we utilized live labeling of common AMPA and NMDA glutamate receptor subunits and analyzed the receptor surface population. In cultured hippocampal neurons transfected with shRNA targeting Zip8, we found a significant reduction of surface glutamate receptor subunits, including GluA1, GluA2/3, GluN1, and GluN2A (Fig. 3A, B). Consistent with the finding that zinc has a 50 -fold lower affinity for GluN2B-containing NMDARs ${ }^{13,29-31}$, we did not observe a change in the surface expression of this receptor subtype (Fig. 3A, B). 
A
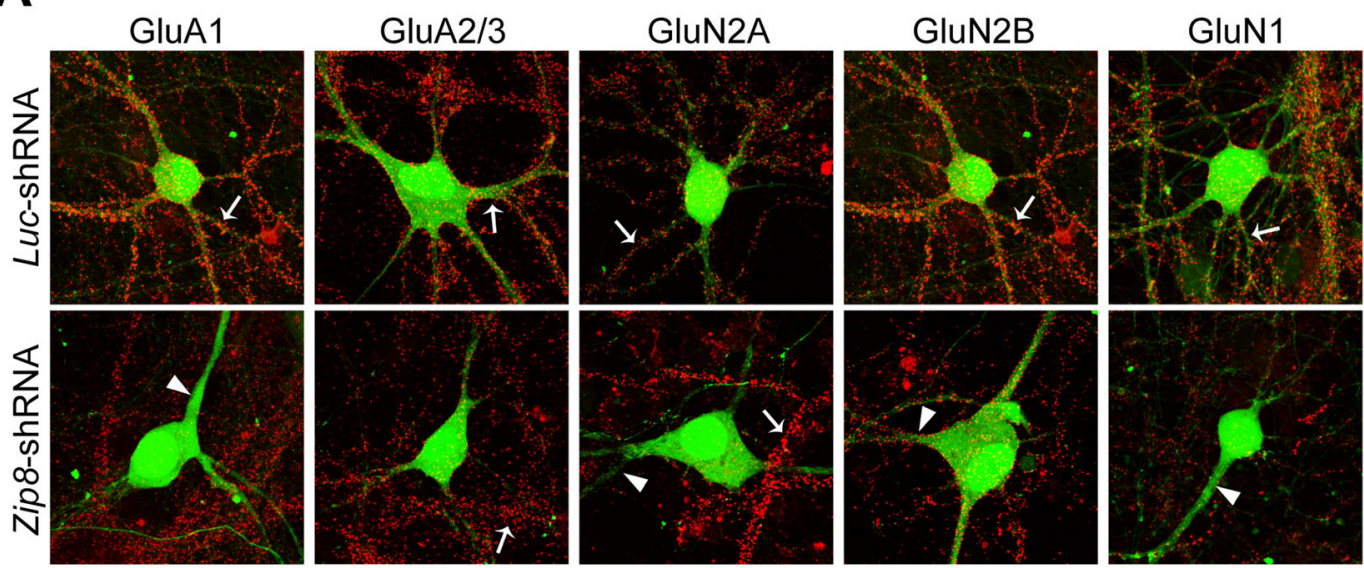

B

C
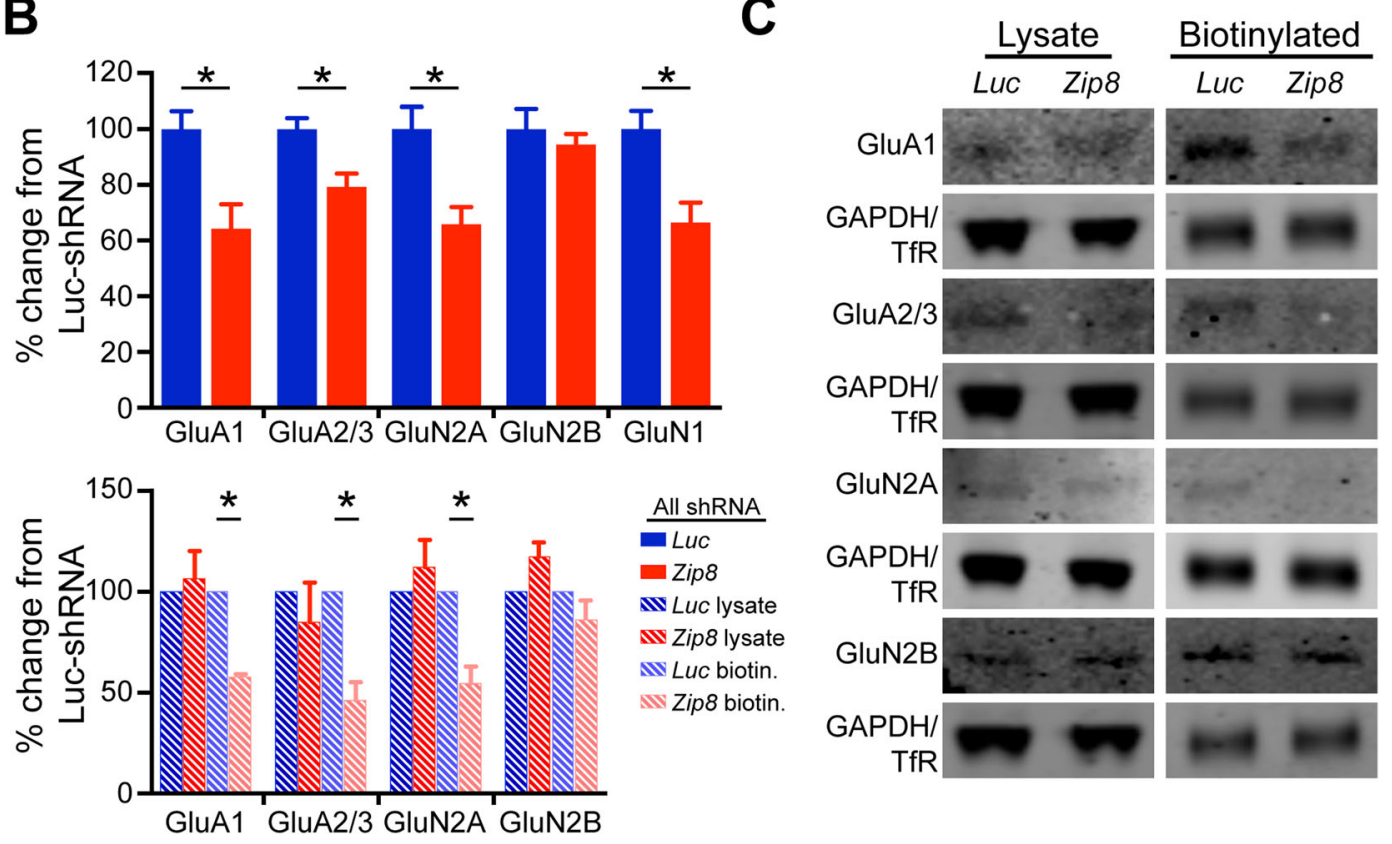

TfR

Fig. 3 ZIP8 KO in cultured neurons leads to decreased surface localization of specific glutamate receptor subunits. A Representative neurons expressing GFP-tagged Luc- or Zip8-shRNA and stained with antibodies against different glutamate receptor subunits at DIV28-30. Arrows denote neurites from Luc-shRNA-expressing neurons (top) or surrounding non-transfected neurons in the Zip8-shRNA condition (bottom). Arrow heads indicate neurites from Zip8-shRNA-expressing neurons. B Quantification of different glutamate receptor subunits' staining intensity relative to LucshRNA treatment. Intensity of signal for each subunit from the Luc-shRNA treatment was averaged and used to normalize Luc-and Zip8-shRNA treatments. ${ }^{*} P<0.05$ (Student's $t$-test); $n=6-10$ per group. Error bar, SEM. C Representative western blot of individual glutamate receptor subunits, as quantified in $\mathbf{B}$.

To confirm our initial findings that knockdown of ZIP8 leads to increased zinc levels and decreased glutamate receptor surface expression, we used a surface biotinylation assay to query the levels of surface versus total glutamate receptor expression under different conditions. Only the biotinylated populations of certain glutamate receptor subunits, including GluA1, GluA2/3, and GluN2A, were decreased in ZIP8 KD neuronal lysates (Fig. 3). Consistent with the live labeling experiments, the GluN2B subunit did not show significant changes in biotinylated levels in ZIP8 KD lysates (Fig. 3C). Importantly, all glutamate receptor subunits had similar levels of total protein amounts between Luc-shRNA- and Zip8-shRNA-treated neurons, demonstrating that the surface levels of specific glutamate receptor subunits that are susceptible to synaptic zinc inhibition underwent dynamic activity-dependent regulation. 


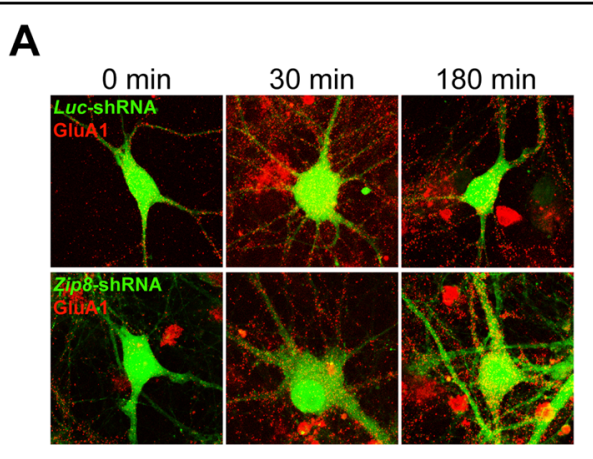

C

Pre ZX1 application

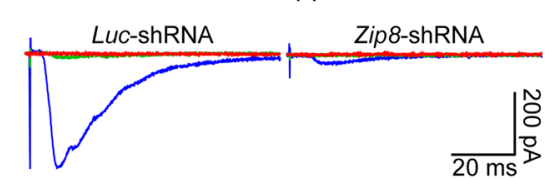

Post ZX1 application

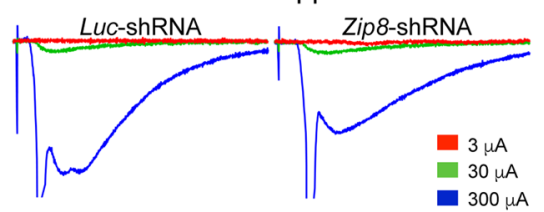

B

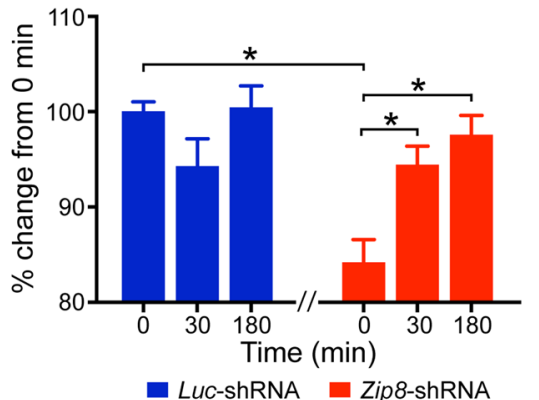

D

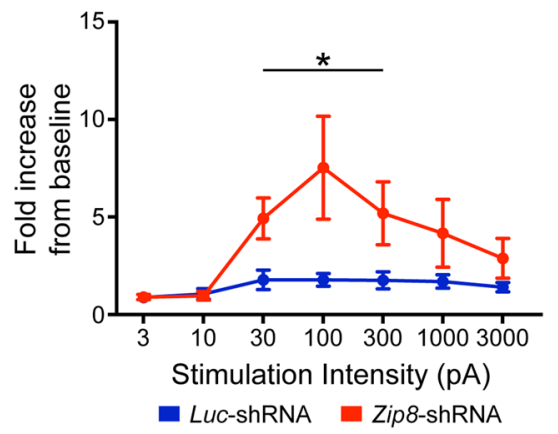

Fig. 4 Zinc chelation rescues GluA1 receptor surface localization and evoked EPSC amplitude in hippocampal CA1 neurons. A Time course of $100 \mu \mathrm{M}$ ZX1 treatment in Luc- or Zip8-shRNA-expressing cultured pyramidal neurons stained with antibodies against the GluA1 subunit. B Quantification of GluA1 puncta over time following ZX1 application. Data are normalized to Luc-shRNA at 0 min. Note the significant difference between treatments prior to zinc chelation (two-way ANOVA followed by Sidak's post hoc test; Luc vs. Zip8 at 0 min $(P<0.001)$, Zip8 0 min vs. 30 min $(P<0.02)$, Zip8 0 min vs. $180 \mathrm{~min}(P<0.04) ; n=6-10$ per group). Error bar, SEM. C Representative traces of Schaffer collateral-evoked EPSCS from hippocampal CA1 neurons transfected in vivo with virus encoding either Luc- or Zip8-shRNA then recorded ex vivo using whole-cell patch clamp. Recordings made from Schaffer collateral stimulation before and after treatment of $Z X 1$ ( $n=6$ per group). Note that the same stimulation intensities given prior to ZX1 now result in a large population event, typical of a loss of zinc-mediated inhibition ${ }^{33,34}$. D Quantification of evoked signals at different stimulation intensities shown to the right (two-way ANOVA followed by Sidak's post hoc test; Luc + ZX1 vs. Zip8 + ZX1 effect of treatment $(P<0.0001))$. Error bars, SEM.

Chelation of extracellular zinc rescues glutamate receptor surface localization and function in ZIP8 knockdown neurons

Given our results suggesting that ZIP8 loss-of-function may lead to the accumulation of free zinc in the synaptic cleft and the subsequent inhibition of glutamate receptors, we next evaluated whether removing the excess zinc would reverse the phenotypes observed in ZIP8 KD neurons. We focused our analyses on GluA1 receptor expression because of its robust expression and thus larger dynamic window for immunocytochemistry. Using ZX1, a potent extracellular zinc chelator impermeable to neurons ${ }^{32}$, we observed a restoration of GluA1 subunits on the surface of ZIP8 KD cells after 30 min of incubation with ZX1, which persisted up through $180 \mathrm{~min}$ of ZX1 preincubation (Fig. 4A, B). This result further suggests that the decreased surface levels of certain glutamate receptor subunits are reversible, as would be expected from the activity-dependent dynamic regulation of receptor surface levels (Figs. 2A, C and S1).
We next examined whether our observations in cultured neurons would translate to the intact brain. One month after injecting lentivirus encoding either Luc-Gfp-shRNA or Zip8-Gfp-shRNA into the hippocampal CA1 region of C57/B6 mice, we recorded the Schaffer collateral-evoked glutamate-mediated excitatory activity from GFP-labeled hippocampal CA1 pyramidal cells. Prior to application of ZX1, control Luc-shRNA-treated CA1 neurons showed higher evoked glutamate receptor activity compared to Zip8-shRNA-treated neurons (Fig. 4C, D), consistent with our results in cultured neurons (Fig. 2). In agreement with the rescue of glutamate receptor surface expression (Fig. 4A), evoked currents in CA1 neurons infected with virus encoding Zip8-shRNA were normalized compared to control neurons (Fig. 4C, D) 30 min after ZX1 application, indicative of removal of all zinc-mediated inhibition ${ }^{33,34}$. Together with our immunofluorescence data, these data are consistent with the suggestion that ZIP8 loss-offunction leads to an accumulation of zinc that inhibits 
synaptic glutamate receptors, which can be reversed by removal of extracellular zinc. These results suggest that ZIP8 is, at least in part, responsible for the dynamic regulation of synaptic glutamate-mediated signaling.

\section{ZIP8 haploinsufficiency leads to an elevated innate immune response in the central nervous system}

While our characterization of the loss-of-function A391T allele suggests that ZIP8 plays a role in regulating glutamate receptor function, it is likely that ZIP8 haploinsufficiency has other deleterious effects, especially given its previously reported role in inflammation. ZIP8 has been reported to affect innate immune functions through coordination of zinc metabolism and inhibition of $\mathrm{NFKB}^{35}$. Interestingly, RNAseq data from Barres and colleagues showed highly enriched ZIP8 expression in brain endothelial cells compared with endothelial cells in peripheral systems ${ }^{36}$, suggesting additional roles of ZIP8 in mediating the immune response in places such as the $\mathrm{BBB}$. Given the evidence that complete $\mathrm{KO}$ of Zip8 in mice is neonatally lethal with severe developmental deficits in growth, organ morphogenesis, and hematopoi$\mathrm{esis}^{37}$, we obtained ZIP8 haploinsufficient mice $\left(\mathrm{Zip}^{+/-}\right)$ (courtesy of Dr. Daniel J. Rader, University of Pennsylvania) to investigate the role of ZIP8 in the central nervous system (CNS). We injected Zip $8^{+/-}$mice or control littermates with fluorescent-labeled dextran to examine the integrity of the BBB after stimulation of the innate immune response with a high dose of LPS. Following LPS injections, we detected significantly higher dextran- 488 signals in the brain lysates of $Z i p 8^{+/-}$mice compared to control littermates (Fig. 5A), indicating that ZIP8 hypomorphic animals displayed a leakier BBB upon inflammation. Using meso-scale discovery assays for various cytokine levels, we then confirmed that several key cytokines, including IL- 6 and IL- $1 \beta$, were elevated in the plasma of $\mathrm{Zip8}^{+/-}$mice compared to control littermates (Figs. 5B and S2). Furthermore, using HEK293 cells stably expressing a luciferase NFKB reporter cassette, we found that shRNA-mediated knockdown of ZIP8 led to higher NFKB activation upon TNF $\alpha$ stimulation as compared to Luc-shRNA transfected cells (Fig. 5C and Table S1). Conversely, overexpression of the ZIP $8^{W T}$ construct decreased $\mathrm{NF}_{K} \mathrm{~B}$ activation compared to the wild-type background, whereas the overexpression of ZIP8 ${ }^{A 391 T}$ did not (Fig. 5C and Table S1). Together, these results demonstrate that ZIP8 deficiency leads to morphological changes at the $\mathrm{BBB}$ and potentially harmful hyperactivation of inflammatory pathways.

\section{Discussion}

Although GWAS studies have yielded insights into the genetic architecture underlying risk for schizophrenia, a molecular understanding of how each perturbation translates into the pathology of the disease is still lacking. In this study, we elucidated the biological significance of a human SNP rs13107325 as a loss-of-function A391T allele in zinc transporter ZIP8. We demonstrated that this loss-of-function leads to glutamate receptor hypofunction that is at least in part due to downregulated surface localization of key synaptic glutamate receptors. Finally, we showed that the ZIP8 ${ }^{\mathrm{A} 391 \mathrm{~T}}$ mutation results in an elevated innate immune response. These findings are consistent with observations from patients with schizophrenia and support the glutamate dysfunction ${ }^{38}$ and immune dysfunction ${ }^{39}$ observed in numerous studies. To the best of our knowledge, our data represent the first characterization of the functional consequence of the A391T allele of ZIP8 in CNS glutamate function and are consistent with immune dysfunction observed with the ZIP8 $^{\mathrm{A} 391 \mathrm{~T}}$ mutation in Crohn's disease ${ }^{40}$.

Taken together, our results align with the glutamate hypofunction hypothesis, a central concept of schizophrenia. This theory arises from observations that (1) a majority of the genes that are associated with an increased risk for schizophrenia can influence the function of NMDARs or related receptor-interacting proteins and signal transduction pathways ${ }^{41,42}$; (2) subjects with schizophrenia show profound deficits in neural signals such as mismatch negativity (MMN), thought to be NMDA dependent ${ }^{43}$ and predictive of conversion from the prodromal state ${ }^{44}$; (3) NMDAR antagonists such as phencyclidine and ketamine produce deficits in MMN and cognition as well as psychomimetic effects in healthy individuals reminiscent of symptoms of schizophrenia ${ }^{45,46}$; and (4) mice with reduced NMDAR expression display behaviors thought to be a reflection of psychomimetic effects, such as increased locomotor activity and rearing ${ }^{47}$. Although there was no previous link between NMDARs and the zinc transporter ZIP8, it is well known that NMDARs, particularly the GluN2A subunit, are highly sensitive to synaptic zinc ${ }^{13,16}$. Once ZIP8 is downregulated in neurons, as in our study with hairpin or with expression of the loss-of-function $Z I P 8^{A 391 T}$ allele, zinc import into the cell mediated by ZIP8 is impaired, and zinc would thus have the opportunity to accumulate in the synaptic cleft. Our results predict that individuals carrying the ZIP8 ${ }^{A 391 T}$ allele would have decreased zinc import and consequently elevated levels of synaptic zinc, ultimately leading to glutamate hypofunction due to increased inhibition and/or decreased surface expression of GluN2A-containing NMDARs and AMPARs. Future studies should focus on the complexities of zinc dynamics in the presence of the ZIP8 ${ }^{A 391 T}$ allele.

Our findings that neuronal KD of ZIP8 as well as replacement with ZIP8 ${ }^{\mathrm{A} 391 \mathrm{~T}}$ results in decreased amplitude of NMDA- and AMPA-mediated sEPSCs likely represent an increase in synaptic zinc due to a decrease in the control of 
A

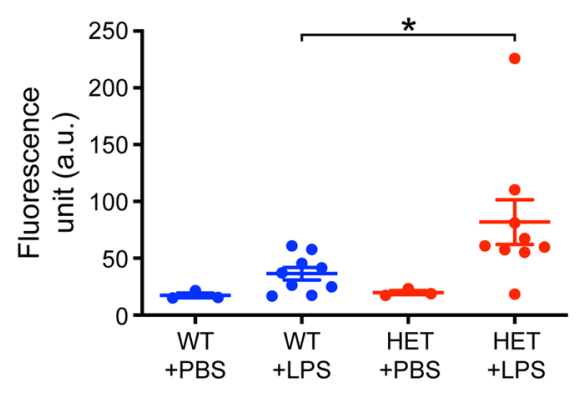

B

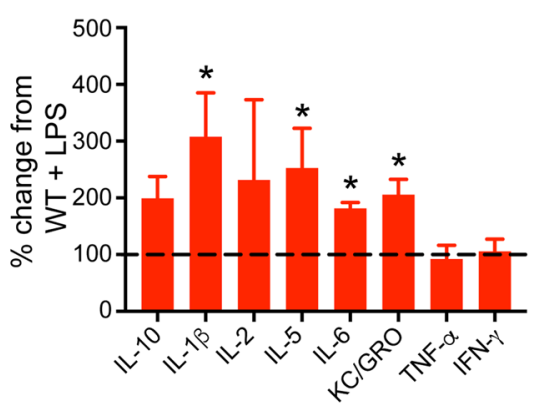

C

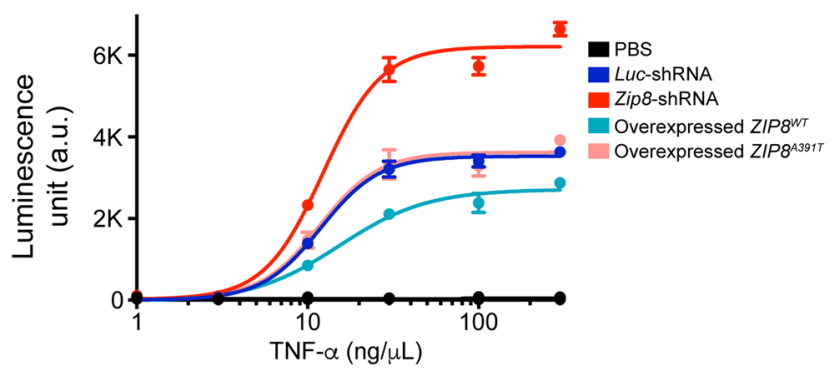

Fig. 5 ZIP8 loss-of-function results in increased BBB leakage and overactivation of NFKB. A Intravenous retro-orbital sinus injection of Dextran488 after $24 \mathrm{~h}$ LPS treatment. Mice that were haplo-insufficient for Zip8 demonstrated an elevated dextran 488 level compared to WT mice. ${ }^{*} P<0.05$ (Student's $t$-test; $n=3$ per PBS-treated group and $n=7-10$ per LPS-treated group). Error bar, SEM. B Zip8 HET mice after $24 \mathrm{~h}$ LPS treatment displayed increased levels of several plasma cytokines compared to WT mice (one-way ANOVA followed by Tukey's multiple comparison test, ${ }^{*}=$ significant difference between WT LPS and HET LPS conditions: KC/GRO, $P=0.042 ;$ IL-5, $P=0.05 ;$ IL-1 $\beta, P=0.05 ;$ IL-6, $P=0.002 ;$ see Fig. S2). Cytokine levels from WT mice treated with LPS were averaged and used for normalizing cytokine response from Zip8 HET mice treated with LPS ( $n=17$ per group). Error bar, SEM. C In HEK293 cells stably expressing a 3xNFKB reporter, Zip8-shRNA transient transfection led to increased NFkB activation compared to LucshRNA transiently transfected cells, whereas cells transfected with ZIP8 ${ }^{W T}$ plasmid displayed a protective effect compared to cells transfected with ZIP8A391T plasmid, which had no effect on the WT background (two-way ANOVA followed by Bonferonni's post hoc test; TNFa vs. manipulation interaction $(P<0.0001)$. See Table S1 for details. Error bar, SEM.

synaptic zinc. This probable increase of zinc in the extrasynaptic space likely dampens NMDAR- and AMPARmediated activity, leaving a modest amount of postsynaptic activity intact as demonstrated in our electrophysiological data (Fig. 2). Notably, the reduction of GluN2A, but not GluN2B, surface expression is consistent with data showing that zinc is many-fold more selective for GluN2A receptors $^{13,29-31,48,49}$ and AMPARs ${ }^{34}$. We found that persistent, but not total, inhibition of these receptors by dysregulation of synaptic zinc leads to receptor internalization of GluN2ANMDARs ${ }^{50}$ and GluA1-AMPARs as suggested by decreased surface expression (Fig. 3), perhaps mimicking a form of long-term depression (LTD) ${ }^{51}$. Our finding that surface expression of GluN2B-containing receptors was not affected by ZIP8 KD or the ZIP8 ${ }^{\mathrm{A} 391 \mathrm{~T}}$ mutation is likely because the $\mathrm{IC}_{50}$ of zinc for these receptor subtypes is at least 50 -fold higher compared to GluN2A-NMDARs ${ }^{13,48,49}$. Prior findings have demonstrated that GluN2B-, but not GluN2A-, containing NMDARs are required for induction of $\operatorname{LTD}^{52,53}$. Therefore, the increased inhibition selectively at GluN2Acontaining NMDARs, leaving GluN2B-mediated NMDA signaling intact, may explain the decrease in GluA1 receptor surface expression observed (Fig. 3), similar to that observed when GluN2A-containing NMDARs are knocked down ${ }^{54}$.

The demonstration that overexpression of ZIP $8^{W T}$ and the chelation of extracellular zinc rescues both receptor surface expression (Fig. 4A-D) as well as function (Figs. 2 and 4) further supports the hypothesis that the ZIP8 ${ }^{\mathrm{A} 391 \mathrm{~T}}$ mutation results in glutamate hypofunction consistent with that observed in schizophrenia. Interestingly, the chelation of zinc over a 30-min period not only rescued evoked EPSCs but exacerbated the population spike amplitude compared to the luciferase condition. This finding is unsurprising, however, as chelation of zinc with ZX1 results in loss of all endogenous zinc content, thus removing any ongoing zinc-mediated inhibition. These data are consistent with the increase in LTP observed following chelation of zinc in hippocampal slices ${ }^{55}$.

Finally, chronic inflammation in the CNS is considered pathogenic and hypothesized to be responsible for many diseases such as multiple sclerosis and schizophrenia ${ }^{56,57}$. In schizophrenia, dysregulation of the immune system is often 
evidenced in the elevation of type- 2 cytokines such as IL-6 and IL-10 (ref. ${ }^{58}$ ). Although zinc is thought to be important in the peripheral innate immune system as an inhibitor of $\mathrm{NF}_{\kappa} \mathrm{B}^{35}$, little is known about the role of either zinc or ZIP8 in regulating immune responses in the CNS. In our study, Zip8 hypomorphic mice displayed deficits in the integrity of the BBB after LPS challenge as shown by a greater dye concentration collected in the brain homogenate following intravenous injection compared to controls. This finding reflects a hallmark condition of CNS inflammation in which a compromised BBB facilitates transmigration of lymphocytes $^{59}$. Blood samples from the Zip8 ${ }^{+/-}$mice, collected after LPS treatment, displayed higher levels of several cytokines, including IL- 6 and IL-10, which have been shown to be elevated in schizophrenia. Our results also showed that overexpressing ZIP8 ${ }^{\mathrm{WT}}$ protein in NFkB reporter cell lines decreased NFKB signaling, whereas overexpressing the $\mathrm{ZIP}^{\mathrm{A} 391 \mathrm{~T}}$ variant did not. This result correlates well with previous observations that ZIP8 KO and A391T allele in cellular systems are linked to an elevated immune response ${ }^{35,40,60}$. Together, these findings implicate CNS inflammation as another avenue by which ZIP8 dysfunction could increase the risk for psychiatric disease. Whether immune system dysregulation or glutamate hypofunction is more central to the ZIP8 ${ }^{\mathrm{A} 391 \mathrm{~T}}$ mutation's risk remains to be elucidated, and future work should be done to better understand the extent to which these different pathways might interact. Future studies should focus on full characterization of behavioral phenotypes in the ZIP8 ${ }^{\mathrm{A} 391 \mathrm{~T}}$ mouse before and after specific manipulation of either CNS activity or inflammation.

Given the reported CNS inflammation in schizophrenia patients $^{22,56}$, ZIP8 hypofunction (as in the context of the A391T allele) could engender psychiatric risk both by leading to glutamate receptor hypofunction as well as increased inflammation. Our data indicate that targeted treatment with mechanisms selectively enhancing glutamate function and/or targeting anti-inflammatory mechanisms may benefit this subpopulation of patients with schizophrenia. Stratifying subjects based on their autoimmune response or using measures sensitive to NMDA-mediated responses to glutamate, such as MMN, may enable greater success for therapeutics aiming to treat schizophrenia.

\section{Acknowledgements}

The authors would like to thank Dr. Charles Anderson (West Virginia University) and Dr. Rita Balice-Gordon (Muna Therapeutics) for their comments and guidance on earlier versions of the manuscript. The authors also acknowledge Kewa Mou (Pfizer) for generating cortical cell cultures for experiments.

\section{Author details}

${ }^{1}$ Wave Life Sciences, Cambridge, MA 02138, USA. ${ }^{2}$ Biogen, Cambridge, MA 02139, USA. ${ }^{3}$ Pfizer, Inc., Cambridge, MA 02139, USA. ${ }^{4}$ Takeda Pharmaceuticals, Cambridge, MA 02139, USA

\section{Author contributions}

W.C.T., V.R., T.A.L., M.L.W., J.P., K.X.V.L., and D.L.B. designed and performed the experiments and analyzed the data. W.C.T., V.B., T.A.L., M.L.W., R.D.B., P.O., and D. L.B. participated in the results discussion and interpretation. W.C.T. and D.L.B. conceived the study, interpreted the results, and wrote the paper.

\section{Conflict of interest}

When this work was performed, each author was a full-time employee of Pfizer Inc. and owned and/or held options/restricted stock units for the company's publicly traded shares.

\section{Publisher's note}

Springer Nature remains neutral with regard to jurisdictional claims in published maps and institutional affiliations.

Supplementary information The online version contains supplementary material available at https://doi.org/10.1038/s41398-021-01262-5.

Received: 11 January 2021 Accepted: 2 February 2021

Published online: 19 February 2021

\section{References}

1. Farrell, M. S. et al. Evaluating historical candidate genes for schizophrenia. Mol. Psychiatry 20, 555-562 (2015).

2. Carrera, N. et al. Association study of nonsynonymous single nucleotide polymorphisms in schizophrenia. Biol. Psychiatry 71, 169-177 (2012).

3. Schizophrenia Working Group of the Psychiatric Genomics, C. Biological insights from 108 schizophrenia-associated genetic loci. Nature $\mathbf{5 1 1}, 421-427$ (2014).

4. Hess, J. L. et al. Transcriptome-wide mega-analyses reveal joint dysregulation of immunologic genes and transcription regulators in brain and blood in schizophrenia. Schizophr. Res. 176, 114-124 (2016).

5. Wockner, L. F. et al. Genome-wide DNA methylation analysis of human brain tissue from schizophrenia patients. Transl. Psychiatry 4, e339 (2014).

6. Dalton, T. P. et al. Identification of mouse SLC39A8 as the transporter responsible for cadmium-induced toxicity in the testis. Proc. Natl Acad. Sci. USA 102, 3401-3406 (2005).

7. He, L. et al. ZIP8, member of the solute-carrier-39 (SLC39) metal-transporter family: characterization of transporter properties. Mol. Pharmacol. 70, 171-180 (2006).

8. Nakashima, A. S. \& Dyck, R. H. Zinc and cortical plasticity. Brain Res. Rev. 59, 347-373 (2009).

9. Bitanihirwe, B. K. \& Cunningham, M. G. Zinc: the brain's dark horse. Synapse $\mathbf{6 3}$, 1029-1049 (2009).

10. Sensi, S. L., Paoletti, P., Bush, A. I. \& Sekler, I. Zinc in the physiology and pathology of the CNS. Nat. Rev. Neurosci. 10, 780-791 (2009).

11. Vogt, K., Mellor, J., Tong, G. \& Nicoll, R. The actions of synaptically released zinc at hippocampal mossy fiber synapses. Neuron 26, 187-196 (2000).

12. Qian, J. \& Noebels, J. L. Visualization of transmitter release with zinc fluorescence detection at the mouse hippocampal mossy fibre synapse. J. Physiol. 566, 747-758 (2005).

13. Paoletti, P., Ascher, P. \& Neyton, J. High-affinity zinc inhibition of NMDA NR1NR2A receptors. J. Neurosci. 17, 5711-5725 (1997).

14. Traynelis, S. F., Burgess, M. F., Zheng, F., Lyuboslavsky, P. \& Powers, J. L. Control of voltage-independent zinc inhibition of NMDA receptors by the NR1 subunit. J. Neurosci. 18, 6163-6175 (1998).

15. Paoletti, P. et al. Molecular organization of a zinc binding n-terminal modulatory domain in a NMDA receptor subunit. Neuron $\mathbf{2 8}, 911-925$ (2000).

16. Vergnano, A. M. et al. Zinc dynamics and action at excitatory synapses. Neuron 82, 1101-1114 (2014).

17. Olney, J. W., Newcomer, J. W. \& Farber, N. B. NMDA receptor hypofunction model of schizophrenia. J. Psychiatr. Res. 33, 523-533 (1999).

18. Homayoun, H. \& Moghaddam, B. NMDA receptor hypofunction produces opposite effects on prefrontal cortex interneurons and pyramidal neurons. J. Neurosci. 27, 11496-11500 (2007). 
19. Najjar, S. et al. Neurovascular unit dysfunction and blood-brain barrier hyperpermeability contribute to schizophrenia neurobiology: a theoretical integration of clinical and experimental evidence. Front. Psychiatry $\mathbf{8}$, 83 (2017).

20. McCoy, T. H. Jr, Pellegrini, A. M. \& Perlis, R. H. Using phenome-wide association to investigate the function of a schizophrenia risk locus at SLC39A8. Transl. Psychiatry 9, 45 (2019).

21. Ribeiro-Santos, A., Lucio Teixeira, A. \& Salgado, J. V. Evidence for an immune role on cognition in schizophrenia: a systematic review. Curr. Neuropharmacol. 12, 273-280 (2014).

22. Muller, N. Inflammation and the glutamate system in schizophrenia: implications for therapeutic targets and drug development. Expert Opin. Ther Targets 12, 1497-1507 (2008).

23. Huang, Z., Zhang, X. A., Bosch, M., Smith, S. J. \& Lippard, S. J. Tris(2-pyridylmethyl)amine (TPA) as a membrane-permeable chelator for interception of biological mobile zinc. Metallomics 5, 648-655 (2013).

24. Lissin, D. V. et al. Activity differentially regulates the surface expression of synaptic AMPA and NMDA glutamate receptors. Proc. Natl Acad. Sci. USA 95, 7097-7102 (1998).

25. Bogart, L. J. \& O'Donnell, P. Multiple long-range inputs evoke NMDA currents in prefrontal cortex fast-spiking interneurons. Neuropsychopharmacology 43 2101-2108 (2018).

26. Shaffer, C. L. et al. Pharmacological evaluation of clinically relevant concentrations of (2R,6R)-hydroxynorketamine. Neuropharmacology 153, 73-81 (2019).

27. Simpkins, K. L. et al. Selective activation induced cleavage of the NR2B subunit by calpain. J. Neurosci. 23, 11322-11331 (2003).

28. Besser, L. et al. Synaptically released zinc triggers metabotropic signaling via a zinc-sensing receptor in the hippocampus. J. Neurosci. 29, 2890-2901 (2009).

29. Chen, N., Moshaver, A. \& Raymond, L. A. Differential sensitivity of recombinant $\mathrm{N}$-methyl-D-aspartate receptor subtypes to zinc inhibition. Mol. Pharmacol. 51, 1015-1023 (1997)

30. Rachline, J., Perin-Dureau, F., Le Goff, A., Neyton, J. \& Paoletti, P. The micromolar zinc-binding domain on the NMDA receptor subunit NR2B. J. Neurosci. 25 308-317 (2005).

31. Karakas, E., Simorowski, N. \& Furukawa, H. Structure of the zinc-bound aminoterminal domain of the NMDA receptor NR2B subunit. EMBO J. 28, 3910-3920 (2009).

32. Pan, E. et al. Vesicular zinc promotes presynaptic and inhibits postsynaptic long-term potentiation of mossy fiber-CA3 synapse. Neuron 71, 1116-1126 (2011).

33. Anderson, C. T. et al. Modulation of extrasynaptic NMDA receptors by synaptic and tonic zinc. Proc. Natl Acad. Sci. USA 112, E2705-E2714 (2015).

34. Kalappa, B. I., Anderson, C. T., Goldberg, J. M., Lippard, S. J. \& Tzounopoulos, T. AMPA receptor inhibition by synaptically released zinc. Proc. Natl Acad. Sci. USA 112, 15749-15754 (2015).

35. Liu, M. J. et al. ZIP8 regulates host defense through zinc-mediated inhibition of NF-kappaB. Cell Rep. 3, 386-400 (2013).

36. Zhang, $Y$. et al. An RNA-sequencing transcriptome and splicing database of glia, neurons, and vascular cells of the cerebral cortex. J. Neurosci. 34, 11929-11947 (2014).

37. Galvez-Peralta, M. et al. ZIP8 zinc transporter: indispensable role for both multiple-organ organogenesis and hematopoiesis in utero. PLOS ONE 7, e36055 (2012).

38. Howes, O., McCutcheon, R. \& Stone, J. Glutamate and dopamine in schizophrenia: an update for the 21st century. J. Psychopharmacol. 29, 97-115 (2015).

39. Leza, J. C. et al. Inflammation in schizophrenia: a question of balance. Neurosci. Biobehav. Rev. 55, 612-626 (2015).
40. Pyle, C. J. et al. Zinc modulates endotoxin-induced human macrophage inflammation through ZIP8 induction and C/EBPbeta inhibition. PLOS ONE 12, e0169531 (2017).

41. Harrison, P. J. \& Weinberger, D. R. Schizophrenia genes, gene expression, and neuropathology: on the matter of their convergence. Mol. Psychiatry 10, 40-68 (2005).

42. Snyder, M. A. \& Gao, W. J. NMDA hypofunction as a convergence point for progression and symptoms of schizophrenia. Front. Cell Neurosci. 7, 31 (2013).

43. Avissar, M. \& Javitt, D. Mismatch negativity: a simple and useful biomarker of $\mathrm{N}$-methyl-d-aspartate receptor (NMDAR)-type glutamate dysfunction in schizophrenia. Schizophr. Res. 191, 1-4 (2018).

44. Addington, J. et al. North American Prodrome Longitudinal Study (NAPLS 2): the prodromal symptoms. J. Nerv. Ment. Dis. 203, 328-335 (2015).

45. Lahti, A. C., Koffel, B., LaPorte, D. \& Tamminga, C. A. Subanesthetic doses of ketamine stimulate psychosis in schizophrenia. Neuropsychopharmacology $\mathbf{1 3}$ 9-19 (1995)

46. Krystal, J. H. et al. Subanesthetic effects of the noncompetitive NMDA antagonist, ketamine, in humans. Psychotomimetic, perceptual, cognitive, and neuroendocrine responses. Arch. Gen. Psychiatry 51, 199-214 (1994).

47. Mohn, A. R., Gainetdinov, R. R., Caron, M. G. \& Koller, B. H. Mice with reduced NMDA receptor expression display behaviors related to schizophrenia. Cell $\mathbf{9 8}$ 427-436 (1999).

48. Williams, K. Separating dual effects of zinc at recombinant N-methyl-Daspartate receptors. Neurosci. Lett. 215, 9-12 (1996).

49. Nozaki, C. et al. Zinc alleviates pain through high-affinity binding to the NMDA receptor NR2A subunit. Nat. Neurosci. 14, 1017-1022 (2011).

50. Zhu, J. et al. Chronic zinc exposure decreases the surface expression of NR2Acontaining NMDA receptors in cultured hippocampal neurons. PLOS ONE 7, e46012 (2012).

51. Beattie, E. C. et al. Regulation of AMPA receptor endocytosis by a signaling mechanism shared with LTD. Nat. Neurosci. 3, 1291-1300 (2000).

52. Dalton, G. L., Wu, D. C., Wang, Y. T., Floresco, S. B. \& Phillips, A. G. NMDA GluN2A and GluN2B receptors play separate roles in the induction of LTP and LTD in the amygdala and in the acquisition and extinction of conditioned fear. Neuropharmacology 62, 797-806 (2012).

53. Yu, S. Y., Wu, D. C. \& Zhan, R. Z. GluN2B subunits of the NMDA receptor contribute to the AMPA receptor internalization during long-term depression in the lateral amygdala of juvenile rats. Neuroscience 171, 1102-1108 (2010).

54. Kim, M. J., Dunah, A. W., Wang, Y. T. \& Sheng, M. Differential roles of NR2A- and NR2B-containing NMDA receptors in Ras-ERK signaling and AMPA receptor trafficking. Neuron 46, 745-760 (2005).

55. Shetty, M. S., Sharma, M. \& Sajikumar, S. Chelation of hippocampal zinc enhances long-term potentiation and synaptic tagging/capture in CA1 pyramidal neurons of aged rats: implications to aging and memory. Aging Cell 16, 136-148 (2017).

56. Muller, N., Weidinger, E., Leitner, B. \& Schwarz, M. J. The role of inflammation in schizophrenia. Front. Neurosci. 9, 372 (2015).

57. Anderson, G. et al. Immuno-inflammatory, oxidative and nitrosative stress, and neuroprogressive pathways in the etiology, course and treatment of schizophrenia. Prog. Neuropsychopharmacol. Biol. Psychiatry 42, 1-4 (2013).

58. Muller, N. \& Schwarz, M. J. Neuroimmune-endocrine crosstalk in schizophrenia and mood disorders. Expert Rev. Neurother. 6, 1017-1038 (2006).

59. Kebir, H. et al. Human TH17 lymphocytes promote blood-brain barrier disruption and central nervous system inflammation. Nat. Med. 13, 1173-1175 (2007).

60. Melia, J. M. P. et al. Induction of the metal transporter ZIP8 by interferon gamma in intestinal epithelial cells: Potential role of metal dyshomeostasis in Crohn's disease. Biochem. Biophys. Res. Commun. 515, 325-331 (2019). 